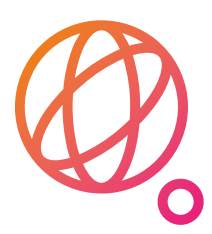

\title{
O.UANTUM
}

FLAGSHIP

\section{EUROPE'S QUANTUM FLAGSHIP IS TAKING OFF}

\author{
- Max F. Riedel ${ }^{1}$, Immanuel Bloch ${ }^{2,3}$, Thierry Debuisschert ${ }^{4}$, Frank Wilhelm-Mauch ${ }^{5}$, Valerio Pruneri ${ }^{6}$, \\ Nikolay V. Vitanov ${ }^{7}$, Stephanie Wehner ${ }^{8}$, Tommaso Calarco ${ }^{9}$ - Dol: https://doi.org/10.1051/epn/2018506 \\ I ${ }^{1}$ Siemens AG - Corporate Technology - Otto-Hahn-Ring 6 - 81739 München, Germany \\ - ${ }^{2}$ Fakultät für Physik - Ludwig-Maximilians-Universität München - Schellingstrasse 4 - 80799 München, Germany \\ [ ${ }^{3}$ Max-Planck-Institut für Quantenoptik, Hans-Kopfermann-Str. 1, 85748 Garching, Germany \\ I ${ }^{4}$ Physics Research Group - Thales Research \& Technology - 1 avenue Augustin Fresnel - 91767 Palaiseau Cedex, France \\ I ${ }^{5}$ Theoretical Physics - Saarland University - 66123 Saarbrücken, Germany \\ I ${ }^{6}$ ICFO - Mediterranean Technology Park - Av. Carl Friedrich Gauss 3 - 08860 Castelldefels (Barcelona), Spain \\ I ${ }^{7}$ Department of Physics - St Kliment Ohridski University of Sofia - 5 James Bourchier Blvd - 1164 Sofia, Bulgaria \\ I ${ }^{8}$ QuTech - Delft University of Technology - Lorentzweg 1 - 2628 CJ Delft, Netherlands \\ - ${ }^{9}$ Forschungszentrum Jülich, Institute of Quantum Control, Peter Grünberg Institut (PGI-8), 52425 Jülich, Germany
}

\section{The European Quantum Flagship is a 10-year, 1 billion Euro initiative, expected to bring quantum technologies from the lab to the market. The initiative is now taking off: The implementation and governance models are decided and the first research projects are commencing their work.}

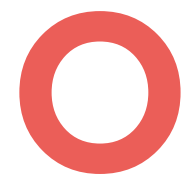
ctober 29 was a big day for quantum technologies (QT) in Europe: In a festive act at the Vienna Hofburg, the Quantum Flagship was officially kicked-off. More than 400 guests, among them distinguished scientists, CEOs of multinational corporations and high-level politicians from all over Europe attended the event. There is good reason for this celebration: The Quantum Flagship is one of the most ambitious research and innovation initiatives of the European Commission, with an expected run-time of 10 years and worth at least one billion Euro of funding money. Its goals are two-fold: On one hand, European scientific leadership and excellence in quantum research, including training the relevant skills, should be consolidated and expanded. On the other hand, the Flagship aims to make Europe a dynamic and attractive region for innovative research, business and investments in QT, thus accelerating their development and take-up by the market and positioning Europe as a leader in the future global commercial QT landscape. To reach these ambitious goals, the Quantum Flagship creates a federated effort from member states and the European Commission, bundling resources to reach a critical mass, competitive with other quantum technology initiatives in the world.

It was a long way to go before the start of the initiative: Europe is not only home to some of the most successful QT research groups, its community is also well connected and organized. A scientific roadmap, written by leaders in their field while taking into account the input of the whole community, was first published in 2005 and has been regularly updated since then (the latest version was published in August 2018 []). In 2016, the European QT community called on the EC to strategically invest in QT as a core future technology. The "Quantum Manifesto" [] was endorsed by over 3500 representatives from academia and industry. The EC decided shortly thereafter to launch the Quantum Flagship initiative, as the third of its kind after the Graphene Flagship and the Human Brain Project.

\section{Implementation and governance}

After announcing the Quantum Flagship, the EC appointed an independent expert group (the High-Level Steering Committee or HLSC), to propose a Strategic Research Agenda (SRA), an implementation structure 
and a governance model for the initiative []. The initial SRA recommended by the HLSC is based on the previously mentioned QT Roadmap, including milestones for the next 3,6 and 10 years.

The Quantum Flagship is implemented through a series of peer-reviewed open calls for research and innovation actions (RIA), making it possible for new ideas and new researchers to contribute throughout the life span of the initiative. They are published as part of H2020 FET and Horizon Europe's Global Challenges pillar, with a foreseen total funding volume of at least $500 \mathrm{M} €$. The EU member states will contribute at least an equal amount through national QT initiatives. A series of coordination and support actions (CSA) accompanies the RIAs to coordinate between the EC and national initiatives, bring academia and industry closer together, and gather input from the QT community to refine and regularly update the SRA. They will also drive standardization and coordinate access to technological infrastructure, such as chip foundries. The CSAs will reach out to the general public and potential end users on behalf of the whole Flagship, and coordinate education and training of a future quantum workforce. Finally, they will act as a coordinator and secretariat for the governance bodies.

The governance of the Quantum Flagship consists of three main elements: The Strategic Advisory Board monitors the progress of the Flagship and advises the EC on strategic measures. It is essentially the successor of the HLSC, chaired by Jürgen Mlynek. Further members will be nominated by the EC in late 2018. The coordinators of the funded projects meet regularly in the Science and Engineering Board to foster knowledge exchange and collaboration between the projects and develop synergies, such as the joint use of facilities. Finally, the Quantum Community Network consists of QT experts from the member states and associated countries, who are well respected and connected both with their national QT community and with their national government. The QCN ensures that input from the broader QT community is taken into account in the Flagship strategy and provides a link to national QT initiatives.

\section{Research and innovation actions of the ramp-up phase}

The first Quantum Flagship call was published in Fall 2017, with a funding budget of $110 \mathrm{M} €$ for RIAs in the areas Quantum Communication, Quantum Computing, Quantum Simulation and Quantum Sensing \& Metrology, an additional $20 \mathrm{M} €$ for Basic Science projects and $2 \mathrm{M} €$ for a CSA. Until the deadline in February 2018, 141 proposals were submitted, bidding for more than $600 \mathrm{M} €$. The evaluation through peer-review resulted in 20 accepted projects, which start on October $1^{\text {st }} 2018$ (see figure 1 and table 1 for details).

\section{Some project examples}

To give the reader a flavour of the scope, size and ambition of the Flagship RIAs, we describe in the following six particular projects in more detail, samples from each area.

\section{QIA - Quantum Internet Alliance (Quantum Communication)}

The Quantum Internet Alliance [] will create a Blueprint for a pan-European Quantum Internet by developing all essential subsystems - quantum repeaters, end nodes as well as the first quantum network stack - culminating in the first experimental demonstration of a fully integrated stack running on a multi-node quantum network.

For this, QIA will push the frontier of technology in both, end nodes (trapped ion qubits, diamond NV qubits, neutral atom qubits) and quantum repeaters (rare-earth-based memories, atomic gases, quantum dots) and demonstrate the first integration of both subsystems. The project will achieve entanglement and teleportation across three and four remote quantum network nodes, thereby making the leap from simple point-to-point connections to first multi-node networks. It will demonstrate the key enabling capabilities for memory-based quantum repeaters, resulting in proof-of-principle demonstrations of elementary long-distance repeater links in the real-world, including the longest such link worldwide.

Hand in hand with hardware development, QIA will realize a software stack that will provide fast, reactive control and allow arbitrary high-level applications to be realized in platform-independent software. QIA's industry partners examine real world use cases of application protocols and their hardware requirements. The project will validate the full stack on a small Quantum Internet by performing an elementary secure quantum cloud computation. QIA will validate the design of the Blueprint architecture by a large-scale simulation of a pan-European Quantum Internet. Through synergy of leading industrial, academic and RTO partners, QIA's Blueprint will set the stage for a strong European Quantum Internet industry.

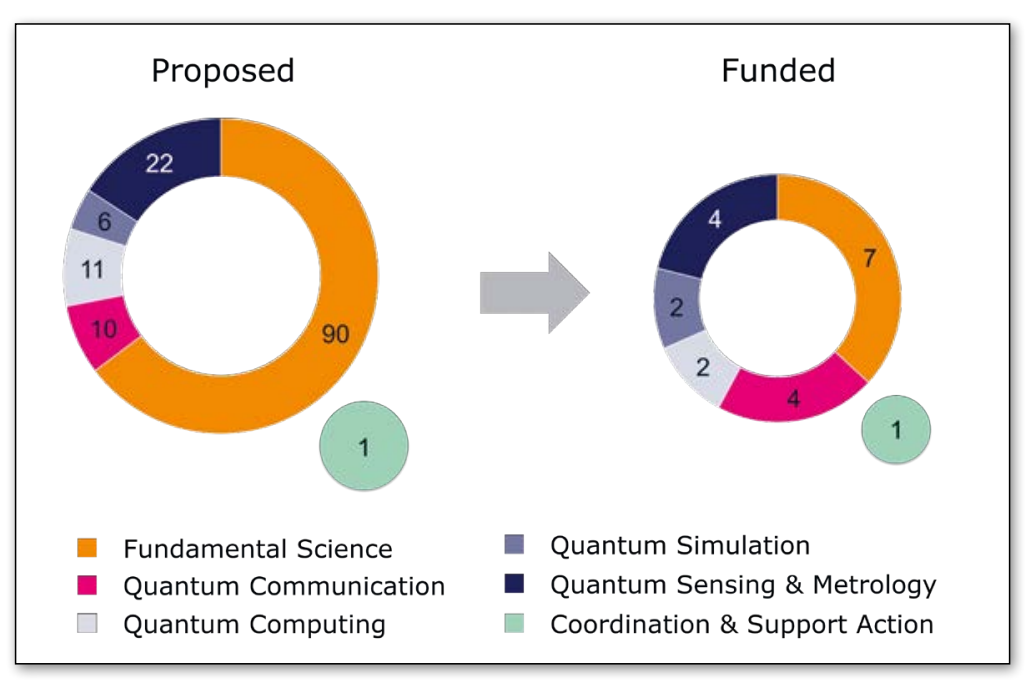

จ FIG. 1:

Number of project proposals (left) and accepted projects (right) per area in the first Quantum Flagship call 
$\checkmark$ TABLE 1: List of accepted projects in the Quantum Flagship ramp-up phase
CiViQ - Continuous Variable Quantum Communication (Quantum Communication) The CiViQ project will aim at developing quantumenhanced physical layer security that can be combined with modern cryptographic techniques, to enable unparalleled applications and services in the teleommmunications arena. It will also put forward novel quantum cryptography systems and protocols, with the ultimate goal of offering accessible, innovative services to individuals, industries and institutions and thus to meet the needs of the secure telecommunications market.

Quantum Key Distribution (QKD) is the most widely used among quantum cryptography protocols. However, its potential for applications in cybersecurity has not yet

\begin{tabular}{|c|c|c|c|}
\hline TITLE & ACRONYM & COORDINATOR NAME & COORDINATOR ORGANISATION \\
\hline \multicolumn{4}{|l|}{ FUNDAMENTAL SCIENCE } \\
\hline $\begin{array}{l}\text { Two-dimensional quantum materials and devices for } \\
\text { scalable integrated photonic circuits }\end{array}$ & 2D-SIPC & Dmitri Efetov & ICF0, Spain \\
\hline Microwave driven ion trap quantum computing & MicroQC & Nikolay Vitanov & $\begin{array}{l}\text { Foundation for Theoretical and Computational Physics } \\
\text { and Astrophysics, Bulgaria }\end{array}$ \\
\hline $\begin{array}{l}\text { Sub-Poissonian Photon Gun by Coherent } \\
\text { Diffusive Photonics }\end{array}$ & PhoG & Natalia Korolkova & $\begin{array}{l}\text { The University Court of the University of St Andrews, } \\
\text { United Kingdom }\end{array}$ \\
\hline Photons for Quantum Simulation & PhoQuS & Alberto Bramati & Sorbonne Université, France \\
\hline Quantum Microwave Communication and Sensing & QMics & Frank Deppe & Bayerische Akademie der Wissenschaften, Germany \\
\hline $\begin{array}{l}\text { Scalable Two-Dimensional Quantum } \\
\text { Integrated Photonics }\end{array}$ & S2QUIP & Klaus Jöns & Kungliga Tekniska Hoegskolan, Sweden \\
\hline Scalable Rare Earth Ion Quantum Computing Nodes & SQUARE & David Hunger & Karlsruher Institut für Technologie, Germany \\
\hline \multicolumn{4}{|l|}{ QUANTUM COMMUNICATION } \\
\hline Continuous Variable Quantum Communications & CiViQ & Valerio Pruneri & ICF0, Spain \\
\hline Quantum Internet Alliance & QIA & Stephanie Wehner & Technische Universiteit Delft, Netherlands \\
\hline $\begin{array}{l}\text { Quantum Random Number Generators: cheaper, faster } \\
\text { and more secure }\end{array}$ & QRANGE & Hugo Zbinden & Université de Genève, Switzerland \\
\hline $\begin{array}{l}\text { Affordable Quantum Communication for Everyone: } \\
\text { Revolutionizing the Quantum Ecosystem from } \\
\text { Fabrication to Application }\end{array}$ & UNIQORN & Hannes Hübel & AIT Austrian Institute of Technology GmbH, Austria \\
\hline \multicolumn{4}{|l|}{ QUANTUM COMPUTING } \\
\hline Advanced quantum computing with trapped ions & AQTION & Thomas Monz & Universität Innsbruck, Austria \\
\hline An Open Superconducting Quantum Computer & OpenSuperQ & Frank Wilhelm-Mauch & Universität des Saarlandes, Germany \\
\hline \multicolumn{4}{|l|}{ QUANTUM SIMULATION } \\
\hline $\begin{array}{l}\text { Programmable Atomic Large-Scale } \\
\text { Quantum Simulation }\end{array}$ & PASQuanS & Immanuel Bloch & $\begin{array}{l}\text { Max-Planck-Gesellschaft zur Forderung der } \\
\text { Wissenschaften eV, Germany }\end{array}$ \\
\hline $\begin{array}{l}\text { Quantum simulation and entanglement engineering in } \\
\text { quantum cascade laser frequency combs }\end{array}$ & Qombs & Augusto Smerzi & Consiglio Nazionale delle Ricerche, Italy \\
\hline \multicolumn{4}{|l|}{ QUANTUM SENSING \& METROLOGY } \\
\hline $\begin{array}{l}\text { Advancing Science and TEchnology thRough dlamond } \\
\text { Quantum Sensing }\end{array}$ & ASTERIQS & Thierry Debuisschert & Thales SA, France \\
\hline Integrated Quantum Clock & iqClock & Florian Schreck & Universiteit van Amsterdam, Netherlands \\
\hline $\begin{array}{l}\text { Miniature Atomic vapor-Cells Quantum devices for } \\
\text { SensIng and Metrology AppLications }\end{array}$ & MACQSIMAL & Jacques Haesler & $\begin{array}{l}\text { CSEM Centre Suisse d'Electronique et de Microtechnique } \\
\text { SA - Recherche et Dévelopement, Switzerland }\end{array}$ \\
\hline $\begin{array}{l}\text { Leveraging room temperature diamond quantum } \\
\text { dynamics to enable safe, first-of-its-kind, multimodal } \\
\text { cardiac imaging }\end{array}$ & MetaboliQs & Christoph Nebel & $\begin{array}{l}\text { Fraunhofer Gesellschaft zur Förderung der } \\
\text { Angewandten Forschung eV, Germany }\end{array}$ \\
\hline \multicolumn{4}{|l|}{ CSA } \\
\hline $\begin{array}{l}\text { Quantum Technology Flagship Coordination and } \\
\text { Support Action }\end{array}$ & QFlag & Markus Wilkens & VDI Technologiezentrum GmbH, Germany \\
\hline
\end{tabular}


been fully exploited, mainly because the systems currently used are often expensive, exhibit poor flexibility, and cannot operate seamlessly in telecommunication networks. CiViQ will overcome the critical valley of death by developing flexible and inexpensive $\mathrm{QKD}$ systems that can be well integrated into telecom infrastructures.

As an alternative to common encoding schemes that use expensive and bulky single photon detection techniques, CiViQ's protocol will measure electric field amplitudes using coherent detection (widely used in modern coherent optical telecommunication). The measured outcomes are continuous values of the amplitudes of electric fields and the detection technique is the ultimate hallmark of CV-QKD (continuous variable), which will lead to the development of inexpensive technology that can be integrated in many more applications, specifically for industrialization and integration in telecommunication networks.

The project brings together a consortium of 21 partners that cover the entire supply chain of QKD, from academic research groups, component manufacturers, industrial equipment suppliers to telecommunication network operators/end users.

\section{OpenSuperQ - An Open Superconducting Quantum Computer (Quantum Computing)}

The collaborative research project OpenSuperQ aims at developing a quantum computing system of up to 100 qubits and to sustainably make it available at a central site for external users. The system consists of a full computing stack: The hardware will be centred on superconducting integrated circuits and contain the necessary technological infrastructure, including a control system and user-friendly cryogenics. The software stack will be integrated from user access all the way to low-level control. The computer will be among the leading platforms in the world and the first of its kind developed in Europe.

The team brings together 11 renowned players from universities, research organisations and businesses covering the fields of science, engineering and application development. The final computer will be located at the world-class supercomputing centre in Jülich, Germany. The core experimental teams build the mission-critical central parts of the quantum computer, while the involved technology partners develop the complex engineering around the device. The theory team supports the experiments by detailed modelling and develops critical applications. Management and dissemination are professionally run by a co-located project management team headed by Professor Frank Wilhelm-Mauch of Saarland University.

One of the unique features of OpenSuperQ is its open and integrative approach, enabling the underlying technologies to serve a large community of users. The central site will allow early adopters to learn how to develop quantum software. The consortium particularly targets applications for quantum simulation in chemistry and materials science as well as for optimisation and machine learning.

\section{PASQUANS - Programmable Atomic Large-Scale Quantum Simulation (Quantum Simulation)}

The PASQuanS project builds on the impressive achievements of the most advanced quantum simulation platforms to date, based on atoms and ions. The neutral-atom simulators handle more than 50 cold atoms in optical lattices or arrays of optical tweezers, interacting via either collisional or Rydberg-state-mediated interactions. The ion-trap platform reaches unsurpassed control with up to 20 ions.

By scaling up these platforms towards $>1000$ atoms or ions, by improving control methods and making these simulators fully programmable, the goal of PASQuanS is to push these already well-advanced platforms far beyond both the state-of-the-art and the reach of classical computation. Full programmability will make it possible to address quantum annealing or optimization problems much sooner than digital quantum computation. One important aspect will be to demonstrate a /quantum advantage for non-trivial problems, paving the way towards practical and industrial applications. PASQuanS will result in modular building blocks for a future generation of quantum simulators.

The PASQuanS project consortium unites five experimental groups with complementary methods to achieve the technological goals, connected with six theoretical teams focusing on certification, control techniques and applications of the programmable platforms, and five industrial partners in charge of the key developments of enabling technologies and possible commercial spinoffs of the project. Possible end-users of these simulators, major industrial actors, are tightly associated with the consortium to help identifying and implementing key applications where quantum simulation provides a competitive advantage.

\section{ASTERIQS - Advancing Science and}

\section{TEchnology thRough dlamond Quantum}

\section{Sensing (Quantum Sensing \& Metrology)}

ASTERIQS [] will exploit quantum sensing based on nitrogen-vacancy-centres in ultrapure diamond to bring solutions to societal and economical needs for which no solution exists yet. Its objectives are to develop:

1. Advanced applications based on magnetic field measurement: a fully integrated scanning diamond magnetometer instrument for nanometer scale measurements, a high dynamics range magnetic field sensor to control advanced batteries used in electrical car industry, a lab-on-chip Nuclear Magnetic Resonance (NMR) detector for early diagnosis of diseases, a 
magnetic field imaging camera for biology or robotics, and an instantaneous spectrum analyser for wireless communications management;

2. New sensing applications to sense temperature within a cell, to monitor new states of matter under high pressure and to sense electric fields with ultimate sensitivity;

3. New measurement tools to elucidate the chemical structure of single molecules by NMR for the pharmaceutical industry or the structure of spintronics devices at the nanoscale for a new generation of spin-based electronic devices.

To achieve these goals, the project will develop enabling tools, such as highest grade diamond material with ultralow impurity level, advanced protocols to overcome residual noise in sensing schemes, and optimized engineering for miniaturized and efficient devices.

ASTERIQS will disseminate its results towards academia and industry and educate the next generation of physicists and engineers. The consortium federates world leading European academic and industrial partners to bring quantum sensing from the laboratory to applications.

MicroQC - Microwave driven ion trap quantum computing (Basic science)

The construction of a large-scale trapped-ion quantum information processor can be made decisively simpler by using the compact microwave technology present already in today's mobile phones and other devices. Microwave technology has tremendous simplification potential by condensing experimental effort down to an engineered conductor microstructure embedded into a chip surface and a few off-the-shelve microwave components. This technology can be the key enabling step for addressing the formidable challenge of a scalable quantum processor for it allows execution of quantum gates by the application of a voltage to a microchip, thereby replacing millions of laser beams, and it can operate at room temperature or mild cooling.

MicroQC aims to demonstrate, through state-of-art quantum engineering, fast and fault-tolerant microwave two-qubit and multi-qubit gates and to design scalable technology components for multi-qubit quantum processors. The successful accomplishment of these objectives, in a combined effort by five leading groups in this field - three experimental groups, including the pioneers in microwave quantum logic with static and oscillating magnetic gradients, and two theory groups - will make large-scale quantum computation and simulation with microwave-controlled microfabricated ion traps possible. Consequently, MicroQC will produce a roadmap to take microwave quantum computation to high technology readiness levels.

\section{Outlook}

The Quantum Flagship is setting sail and we are excited to see this high commitment from all European stakeholders. We find it important to note that quantum technologies will be fostered also beyond the Quantum Flagship through different European funding instruments, possibly including the QuantERA co-fund, the space programme, through the European Research and Innovation Councils and Marie-Skłodowska-Curie-Actions, and the Digital Europe Programme. Concrete steps are already taken: The next QuantERA call will be published in November 2018, a call for a Quantum Key Distribution (QKD) test bed is currently running as part of the H2020 cybersecurity strategy [], and ESA is exploring space-based QKD in its Artes-ScyLight programme []. The German QT initiative was recently announced and further national QT initiatives are expected to be coming soon. The Quantum Flagship thus is really becoming one ship in a larger "quantum fleet".

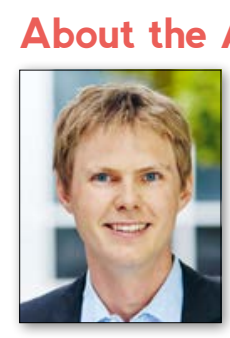

Authors

Max Riedel holds a $\mathrm{PhD}$ in quantum physics and is a Senior Consultant for University Relations at Siemens AG. From January 2017 to September 2018 he was delegated to the University of Ulm as project manager for European activities to support Tommaso Calarco in the preparation of the Quantum Flagship.

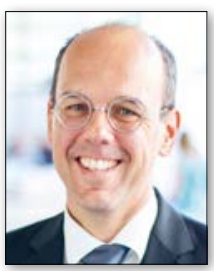

Tommaso Calarco is the director of the Institute Quantum Control (PGI 8 ) at Forschungszentrum Jülich, where he develops control techniques for many-particle quantum systems in order to make quantum technologies more efficient. For more than a decade, he has been instrumental in coordinating the European quantum technology community and is considered one of the intellectual fathers of the Quantum Flagship. Photo credit: Helmholtz/Stefanie Herbst

\section{References}

[1] Antonio Acín et al., New J. Phys. 20, 080201 (2018)

[2] Quantum Manifesto, 2016. Available at: https://qt.eu/resources/

[3] High-Level Steering Committee Report, 2017. Available at: http://ec.europa.eu/newsroom/dae/ document.cfm?doc_id=46979

[4] More information on QIA: http://quantum-internet.team

[5] More information on ASTERIQS: https://www.asteriqs.eu

[6] SU-ICT-04-2019: Quantum Key Distribution testbed

[7] More information on Artes ScyLight: https://artes.esa.int/ artes-scylight-secure-and-laser-communication-technology 